\title{
RHOMBOID FLAP FOR PILONIDAL SINUS- OUR EXPERIENCE
}

\author{
Honeypalsinh H. Maharaul1, Ronak Shah², Mehul Panchal ${ }^{3}$, Darshan Gajera ${ }^{4}$ \\ ${ }^{1}$ Assistant Professor, Department of Surgery, Sumandeep Vidyapeeth, Pipariya, Vododara. \\ ${ }^{2}$ Resident, Department of Surgery, Sumandeep Vidyapeeth, Pipariya, Vododara. \\ ${ }^{3}$ Assistant Professor, Department of Surgery, Sumandeep Vidyapeeth, Pipariya, Vododara. \\ ${ }^{4}$ Resident, Department of Surgery, Sumandeep Vidyapeeth, Pipariya, Vododara.
}

\begin{abstract}
BACKGROUND
Pilonidal sinus is a chronic disease of the sacrococcygeal region. Treatment varies according to the clinical presentation of the disease. Although, many surgical methods have been suggested, an ideal method is still lacking because of high recurrence rates. Recurrent disease causes significant morbidity, particularly missing time from work.

The aim of this study was to assess the rhomboid flap's role in promoting primary healing in recurrent pilonidal disease and to evaluate morbidity and recurrence.
\end{abstract}

ABSTRACT

\section{MATERIALS AND METHODS}

This prospective study included 30 patients who were treated with the use of a rhomboid flap closure for recurrent pilonidal sinus at Dhiraj Hospital from December 2015 to August 2017.

\section{RESULTS}

There were 26 males and 4 females. The mean hospital stay was 9 days (range $=6-12$ days).

\section{CONCLUSION}

Rhomboid flap for pilonidal sinus is suitable for cases where simpler operations have failed. It allows early return to full activity, does not necessitate prolonged postoperative care and has very low recurrence and complication rates which may outweigh the disadvantages related to an unfavourable cosmetic appearance.

\section{KEYWORDS}

Pilonidal Sinus, Rhomboid Flap.

HOW TO CITE THIS ARTICLE: Maharaul HH, Shah R, Panchal M, et al. Rhomboid flap for pilonidal sinus- our experience. J. Evolution Med. Dent. Sci. 2018;7(05):574-577, DOI: 10.14260/jemds/2018/131

\section{BACKGROUND}

Pilonidal sinus is a chronic disease that mostly affects young adults. ${ }^{1}$ This pathology was first described in 1833 and its denomination arises from the Latin terms "pilus" and "nidus" meaning "a nest of hair." Its incidence varies from 10 to 26 per 100,000 population.2,3 Pilonidal disease can be considered an acquired disease, even though the aetiopathology is still not well defined. Pilonidal disease represents several forms of a single abnormality. Pilonidal disease may consist of nothing more than asymptomatic midline skin pits overlying the sacrococcygeal area. At the other extreme, the disease may be a large complex abscess associated with sepsis. In between these extremes are sinuses which occasionally cause discomfort and erythema or sinuses which periodically drain and then return to an asymptomatic state. Some aetiologic factors such as hirsutism, deep natal cleft, obesity, local trauma, familial predisposition, smoking and sedentary lifestyle have been suggested. ${ }^{4,5}$ In our part of country it is common in jeep drivers, so also called as "Jeep's Disease."

'Financial or Other Competing Interest': None.

Submission 22-12-2017, Peer Review 14-01-2018,

Acceptance 22-01-2018, Published 29-01-2018.

Corresponding Author:

Dr. Ronak Shah,

A-41, Omnagar Opp.

Gangasagar Apartment,

Tarsali, Vadodara-390009.

E-mail: ronakshah112@gmail.com

DOI: $10.14260 /$ jemds $/ 2018 / 131$
These epidermal rests can mature, grow and lead to symptoms. In the acquired scenario after the onset of puberty, sex hormones act on the pilosebaceous glands causing the hair follicles to become distended with keratin. As a result, a folliculitis is created which produces oedema and follicle occlusion. The infected follicle ruptures and extends into the subcutaneous tissue. This results in a sinus tract that leads to a deep subcutaneous cavity. By friction or movement of the buttocks with standing or sitting, the distal ends of loose hair from around the perineum may enter these sinuses. The sinuses can then become infected by skin organisms and begin to suppurate, leading to an abscess. Staphylococci and streptococci are the most common organisms found in the culture of infected abscesses.

The incidence of pilonidal disease is approximately $0.7 \%$ in the general population. The condition most commonly affects post-pubertal young adults with males affected two to four times more frequently than females. The onset of the disease occurs earlier in women, which may be due to an earlier onset of puberty. Other factors affecting the incidence are increased sweating associated with sitting and buttock friction, poor personal hygiene, obesity and local trauma. Interestingly, barbers and animal groomers have a higher incidence of the disease than other occupations.

Surgery is the main treatment, and up to $40 \%$ of patients develop recurrence. The management of patients with recurrent disease has led to the development of different surgical approaches. There is no agreement on any optimal surgical technique that would minimise the recurrence rate and controversies are still common.6,7 
The surgical treatments of primary pilonidal sinus include a wide spectrum of techniques that vary from sinus excision with secondary healing of the surgical wound or marsupialisation to the use of flap reconstruction. ${ }^{7}$ Primary closure is preferred in patients with pathology limited to the midline and for whom residual defect after excision is narrow: Karydakis or Bascom procedure can be used. When the residual defect is anticipated to be wide, excision with secondary healing of the wound or flap reconstructions are preferred. ${ }^{3}$ The management of the patient with recurrent disease may require a more complex surgical approach as the excision may be wider or the initial surgery may have led to the loss of the intergluteal tissue. ${ }^{8,9}$

\section{MATERIALS AND METHODS}

This is a prospective study. 30 patients operated on between December 2015 and August 2017 were enrolled in this study. 19 of these patients had a history of previous operations elsewhere. The demographic information analysed included patient's age, sex, familial history of the disease, operation performed for the recurrent disease and duration of followup. The patients selected for the study were contacted by telephone and then called back for clinical evaluation. Patients were asked about pilonidal sinus-related factors including postoperative wound care, duration between operation and recurrence, number of previous operations, type of previous operations and operation type performed for the last episode of the disease. All the operations were performed in the same centre by 2 experienced surgeons.

All patients were operated under spinal anaesthesia in prone position after taking written and informed consent. First incision is marked with the help of ruler and marker (sterile). Incision is kept (Figure 1) and diseased portion is removed and flap is taken to cover raw area and sutured with negative suction in place with non-absorbable suture (polyamide black or polypropylene).

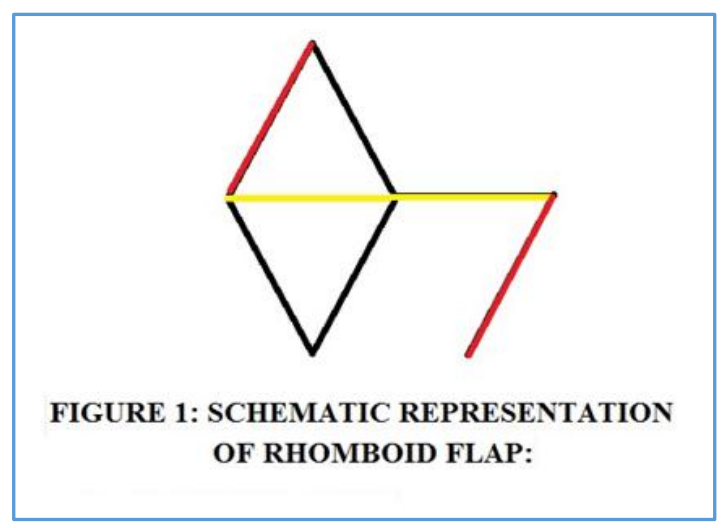

\section{RESULTS}

Out of 30 patients $26(86.6 \%)$ were males and $4(13.34 \%)$ were females (Figure 2). The mean age was 26.3 years. 21 (70\%) patients had a familial history of pilonidal disease. In 27 patients, aetiology-related risk factors (hirsute body habitus, deep natal cleft, obesity, local trauma, familial history, sedentary lifestyle and smoking) were present. Seven (23.34\%) patients had previously been operated on several times before being admitted to our institution. All the patients underwent excision and rhomboid flap surgery (Figure 3). Post-operatively, patients were given antibiotics and analgesics. Dressing was done on day 3 and 5. Drain was removed on day 3. Sutures were removed on days 7 - 12 (depending on healing). Patients were discharged after suture removal and followed up for 6 months.

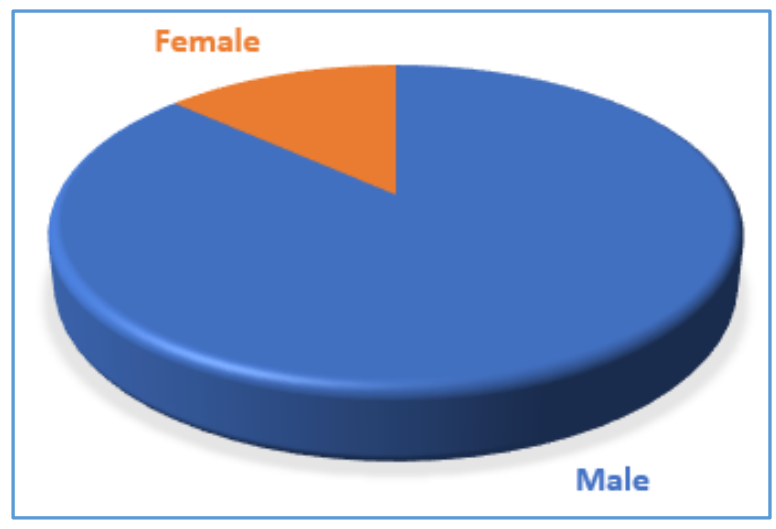

Figure 2. Sex Distribution

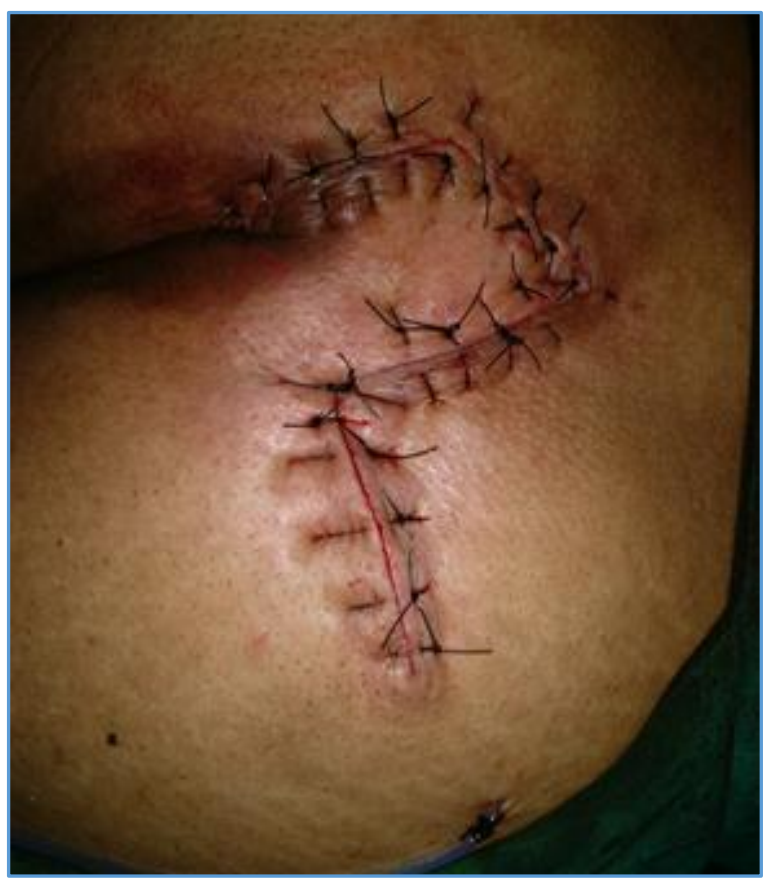

Figure 3. Post-Operative Image

\section{DISCUSSION}

Although, pilonidal sinus cannot be considered a debilitating disease, patients are confronted with discomfort and low quality of life owing to complications such as abscess formation or sinus-related drainage or pain.10,11 The recurrence is most often due to the omission of any tract during the initial operation, infection of the wound or abscess formation that may lead to formation of a new sinus tract inside the cicatrising wound. Accumulation of dead tissue or debris in the intergluteal cleft, sweating, friction or poor hygiene are predisposing factors for recurrence. ${ }^{12}$ In addition, placing the suture line on the midline with considerable tension on the line and failure to lessen the depth of the natal cleft are also important contributors for recurrence. Last, poor wound care with lack of depilation around the wound also contributes to recurrent disease for patients with lay open. ${ }^{13}$

A variety of surgical techniques have been described in the literature for treatment of the primary pilonidal sinus. 
The treatment methods can basically be divided into 2 groups including open and close methods: In the open method, the residual cavity is left open after sinus excision. Lay open is one of the open methods that has also been described and consists of opening the skin covering the sinus and curettage of the residual cavity. This provides a narrower cavity and minimises tissue loss. ${ }^{14}$ Close methods consist of closure of the wound after sinus excision. In this group, the surgical techniques differ according to the type of the wound closure. The cavity can be managed by a midline closure, an offmidline closure or a flap reconstruction.

The open surgical technique is simple to perform. Its recurrence rate is low, but it requires a longer time for wound healing and postoperative wound care. Daily painful wound care and the slow process of secondary healing are the main disadvantages of open techniques, namely lay open.

Lee et $\mathrm{l}^{15}$ recommended primary closure be used in the treatment of the primary disease, but that recurrences should be treated by flap reconstruction. Lieto et al ${ }^{16}$ used sinus excision with flap reconstruction technique in a study on 55 patients with recurrent disease, and only one patient $(1.8 \%)$ developed recurrence. This patient was then successfully treated with the open technique. Better cosmetic results were reported in patients with the primary or secondary disease treated by rhomboid excision with Limberg-Dufourmentel flap reconstruction. Authors report that obesity is an important predisposing factor of recurrence. One crucial point stressed for preventing postoperative recurrence is flattening of the natal cleft and moving the suture line laterally from the midline. Setting out from this idea, Karydakis and Bascom techniques were developed. ${ }^{16,17}$ Majeski et al ${ }^{18}$ treated 127 patients with Bascom technique and reported recurrent disease in 3 patients at 1-year follow-up or sooner. Furthermore, they treated those 3 patients again with Bascom technique successfully and had an excellent cure rate with sutures away from midline. Using the same principle modified Limberg flapping enables tension-free closure, leaving the inferior edge of the incision lateral to the midline. In the prospective study of Mahdy et al, excision and flap reconstruction and excision and primary closure techniques were compared. According to the authors, flap reconstruction was found superior to the primary closure by means of patient comfort and wound healing. Among the flapping techniques, Limberg flapping was found to produce the best results. ${ }^{13}$ Onder et $\mathrm{al}^{19}$ used the Limberg flap and primary closure method in 144 patients in their prospective study. Postoperative complication rates were higher in the flap closure group than in the primary closure method, but recurrence rates were lower. In this present study sinus excision with flap reconstruction was performed in 11 patients, while the open technique after excision was performed in 30 patients. The recurrence rate for flap reconstruction was $9 \%$ (1 patient) and $10 \%$ ( 3 patients) for the open technique. Personal hygiene, periodic epilation and daily wound care are the keystones for preventing recurrence, especially for the patients in the secondary healing group. Thus, proper patient education both in the preoperative and immediate postoperative period is crucial. Close follow-up of the wound is advised and should be carried out in the same centre to facilitate wound evaluation and ultimately lower the recurrence rates.
Some aetiologic factors such as hirsute body habitus, obesity, local trauma, familial predisposition, smoking and sedentary lifestyle have been suggested as predisposing in the development of the primary disease. Predisposing factors are of great importance in the formation of pilonidal sinus disease, which is known to be an acquired disease. These factors also play an important role for recurrent disease. Deep natal cleft, sweating, presence of free hair and poor hygiene with the help of the vacuum created by the hip movements contribute to the formation of new hair cores in the scar tissue. Aetiology-related risk factors (hirsute body habitus, obesity, local trauma, familial history, sedentary lifestyle and smoking) were found in 22 patients for this present study.

Further comparative studies are needed with larger series of patients.

\section{CONCLUSION}

Rhomboid flap for pilonidal sinus is suitable for cases where simpler operations have failed. It allows early return to full activity, does not necessitate prolonged postoperative care and has very low recurrence and complication rates which may outweigh the disadvantages related to an unfavourable cosmetic appearance.

\section{REFERENCES}

[1] Spivak H, Brooks VL, Nussbaum M, et al. Treatment of chronic pilonidal disease. Dis Colon Rectum 1996;39(10):1136-9.

[2] Sondenaa K, Anderson E, Nesvik I, et al. Patient characteristics and symptoms in chronic pilonidal sinus disease. Int J Colorectal Dis 1995;10(1):39-42.

[3] McCallum IJD, King PM, Bruce J. Healing by primary closure versus open healing after surgery for pilonidal sinus: systematic review and metaanalysis. BMJ 2008;336(7649):868-71.

[4] Sondenaa K, Andersen E, Soreide JA. Morbidity and short-term results in a randomised controlled trial of open compared to closed treatment of chronic pilonidal sinus. Eur J Surg 1992;158(6-7):351-5.

[5] Doll D, Matevossian E, Wietelmann K, et al. Family history of pilonidal sinus predisposes to earlier onset of disease and a $50 \%$ long-term recurrence rate. Dis Colon Rectum 2009;52(9):1610-5.

[6] Allen-Mersh TG. Pilonidal sinus: finding the right track for treatment. Br J Surg 1990;77(2):123-2.

[7] Muller K, Marti L, Tarantino I, et al. Prospective analysis of cosmesis, morbidity and patient satisfaction following Limberg flap for the treatment of sacrococcygeal pilonidal sinus. Dis Colon Rectum 2011;54(4):487-94.

[8] Trent JT, Kirsner RS. Wounds and malignancy. Adv Skin Wound Care 2003;16(1):31-4.

[9] Solla JA, Rothenberger DA. Chronic pilonidal disease. An assessment of 150 cases. Dis Colon Rectum 1990;33(9):758-61.

[10] Sondenaa K, Pollard ML. Histology of chronic pilonidal sinus. APMIS 1995;103(4):267-72. 
[11] Lorant T, Ribbe I, Mahteme H, et al. Sinus excision and primary closure versus laying open in pilonidal disease: a prospective randomized trial. Dis Colon Rectum 2011;54(3):300-5.

[12] El-Khadrawy O, Hashish M, Ismail K, et al. Outcome of the rhomboid flap for recurrent pilonidal disease. World J Surg 2009;33(5):1064-8.

[13] Mahdy T. Surgical treatment of the pilonidal disease: primary closure or flap reconstruction after excision. Dis Colon Rectum 2008;51(12):1816-22.

[14] Gençosmanoglu R, Inceoglu R. Modified lay-open (incision, curettage, partial lateral wall excision and marsupialization) versus total excision with primary closure in the treatment of chronic sacrococcygeal pilonidal sinus: a prospective, randomized clinical trial with a complete two-year follow-up. Int J Colorectal Dis 2005;20(5):415-22.
[15] Lee PJ, Raniga S, Biyani DK, et al. Sacrococcygeal pilonidal disease. Colorectal Dis 2008;10(7):639-50.

[16] Karydakis GE. Easy and successful treatment of pilonidal sinus after explanation of its causative process. Aust N Z J Surg 1992;62(5):385-9.

[17] Bascom J. Pilonidal disease: origin from follicles of hairs and results of follicle removal as treatment. Surgery 1980;87(5):567-72.

[18] Majeski J, Stroud J. Sacrococcygeal pilonidal disease. Int Surg 2011;96(2):144-7.

[19] Onder A, Girgin S, Kapan M, et al. Pilonidal sinus disease: risk factors for postoperative complications and recurrence. Int Surg 2012;97(3):224-9. 